Proceedings of the 7-th National Meeting of Synchrotron Radiation Users

\title{
Conventional and Synchrotron X-Ray Topography of Defects in the Core Region of $\mathrm{SrLaGaO}_{4}$
}

A. Malinowska ${ }^{a, b, *}$, M. Lefeld-Sosnowska ${ }^{c}$, K. Wieteska ${ }^{d}$, W. WierzchoWski ${ }^{b}$, A. PAJA̧CZKOWSKA ${ }^{b}$

AND W. GRAEFF ${ }^{e}$

${ }^{a}$ Faculty of Physics, Warsaw University of Technology

Koszykowa 75, PL-00-662 Warsaw, Poland

${ }^{b}$ Institute of Electronic Materials Technology

Wólczyńska 133, PL-01-919 Warsaw, Poland

${ }^{c}$ Institute of Experimental Physics, University of Warsaw

Hoża 69, PL-00-681 Warsaw, Poland

${ }^{d}$ Institute of Atomic Energy, PL-05-400 Otwock-Świerk, Poland

${ }^{e}$ HASYLAB at DESY, Notkestr. 85, D-22603 Hamburg, Germany

$\mathrm{SrLaGaO}_{4}$ single crystals are perspective substrate materials for high temperature superconductors thin films, elements of thermal radiation receivers and other electronic devices. The defect structure of the Czochralski grown $\mathrm{SrLaGaO}_{4}$ crystal was investigated by means of X-ray topography exploring both conventional and synchrotron sources. The crystal lattice defects in the core region of the crystal were investigated. The regular network of defects arranged in rows only in $\langle 100\rangle$ direction was observed. Owing to high resolution of synchrotron radiation white beam back reflection topographs one can distinguish individual spots forming the lines of the rows. It can be supposed that these elongated rod-like volume defects are located in $\{100\}$ lattice planes forming a kind of walls. They are built approximately of the same phase as crystal but crystallize at a different moment than a rest of the crystal due to the constitutional supercooling.

PACS numbers: 61.72.Ff, 61.72.Nn, 61.72.Lk, 61.72.Qq

\section{Introduction}

Oxide materials of general composition $\mathrm{ABCO}_{4}$ (where $\mathrm{A}=\mathrm{Ca}, \mathrm{Sr}, \mathrm{Ba}$, $\mathrm{B}=\mathrm{La}, \mathrm{Nd}, \mathrm{Pr}$ and $\mathrm{C}=\mathrm{Al}, \mathrm{Ga}$ ) with tetragonal perovskite-type structure are perspective substrate materials for high temperature superconductors (HTSc) thin films, elements of thermal radiation receivers and other electronic devices due to

*corresponding author; e-mail: malinows@if.pw.edu.pl 
their electrochemical and thermal properties and good lattice matching as well. It is shown that the most promising material from this group is $\mathrm{SrLaGaO}_{4}$ (SLG) (see Ref. [1] and references therein).

Properties of SLG crystals (for instance, their color) depend on the conditions of growth, for example on the oxygen content in nitrogen atmosphere $[2,3]$. The crystals of good quality were grown in the [100] direction [4-7]. However, the growth of SLG crystals in the [001] direction with the (001) flat interface, was impossible under equilibrium oxygen pressure. The crystals with flat interfaces were also grown on $\{101\}$-type faces. At higher oxygen pressure (over equilibrium) the growth of SLG with flat (001) interface (in the core region) was possible, however, it was accompanied by several defects, some of them possibly associated with local growth surface instabilities.

This situation was explained by the different distribution of ions at (001) and (101) crystal planes and the attachment energies related to the effective charge on oxygen $[1,7]$. Up to now the structure of growth defects in these crystals was not described.

The aim of the present paper is the examination of crystal lattice perfection and mechanisms of the formation of defects in SLG single crystals grown by the Czochralski method in [001] direction. The SLG single crystal grown in the [001] direction in $\mathrm{N}_{2}$ atmosphere with $7.5 \times 10^{3}$ ppm $\mathrm{O}_{2}$ was investigated mainly by means of X-ray topographic methods. The results of our previous studies concerning SLG crystals were shown in Refs. [8-15].

\section{Sample and experimental procedure}

The sample was cut out perpendicular to the growth axis from near seed part of the SLG crystal and mechanically polished to the thickness of $502 \mu \mathrm{m}$. The investigations were performed by X-ray topography exploring both conventional and synchrotron sources of X-rays. The conventional projection X-ray topographs were taken in the reflections from different crystallographic planes in transmission and back reflection geometry using Mo $K_{\alpha_{1}}$ radiation. The synchrotron experiments were realized at stations F1 (white beam) and E2 (monochromatic beam) at HASYLAB (DESY, Hamburg) in back reflection geometry for several crystal azimuths.

In case of white beam synchrotron radiation topography a single exposure provides a large number of topographs corresponding to different Laue spots in one film. The samples were also studied by taking monochromatic beam topographs using synchrotron radiation of $0.115 \mathrm{~nm}$ wavelength. The beam was formed by successive 333 and 511 symmetrical reflections from silicon crystals. In all reproduced topographs dark tones correspond to high X-ray intensity.

\section{Results and discussion}

X-ray topographic images of the investigated sample revealed two regions: a central part - core, which was more perfect and an outer part very well separated 
from the core (Fig. 1a). The same result was observed for another samples studied earlier [8-15]. In the outer part of these crystals a lot of linear black diffraction contrasts and volume defects contrasts of irregular shapes were observed. They are probably associated with facetted growth. The images of the core region revealed linear diffraction contrasts arranged in $\langle 100\rangle$ and $\langle 110\rangle$ directions [9-15].

The crystal core of the sample investigated in this work is well separated from the outer part of the crystal by circular diffraction contrast around the core boundary and by a zone of a perfect crystal (marked by A in Fig. 1a).

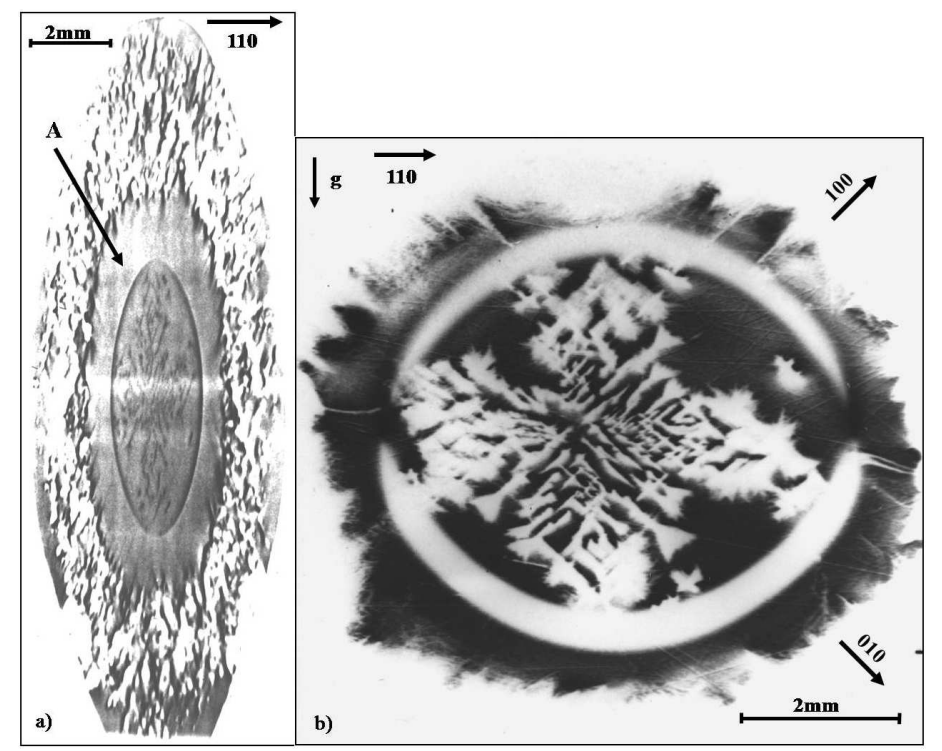

Fig. 1. (a) X-ray back reflection projection topograph, Mo $K_{\alpha_{1}}$ radiation, 0,0,12 reflection; (b) X-ray transmission projection topograph, $\overline{1} 10$ reflection; $\boldsymbol{g}$ - projection of the diffraction vector on the film, $\mu t=16.3$, ( $\mu$ - linear absorption coefficient, $t-$ the crystal thickness).

A regular network of defects arranged in rows in $\langle 100\rangle$-type directions is seen in the core (Fig. 1b). The diffraction contrasts of these defects appear as white lines - in transmission topographs (Fig. 1b) — or black lines in back reflection topographs (Fig. 1a). Very distinct images of these lines are observed in back-reflection synchrotron radiation topographs (Fig. 2). The topographs were obtained for two directions of incoming synchrotron radiation (SR) beam: parallel to the [010] direction in the crystal (Fig. 2a) and parallel to the [100] one (Fig. 2b). One can notice the changes of the diffraction contrast of individual defects. It is the highest for the beam direction perpendicular to the row direction. The difference is very clear in Figs. 2a and b, where the images of the selected defect are shown. One can conclude that the strains are greater in perpendicular direction to the row than in the parallel one. 


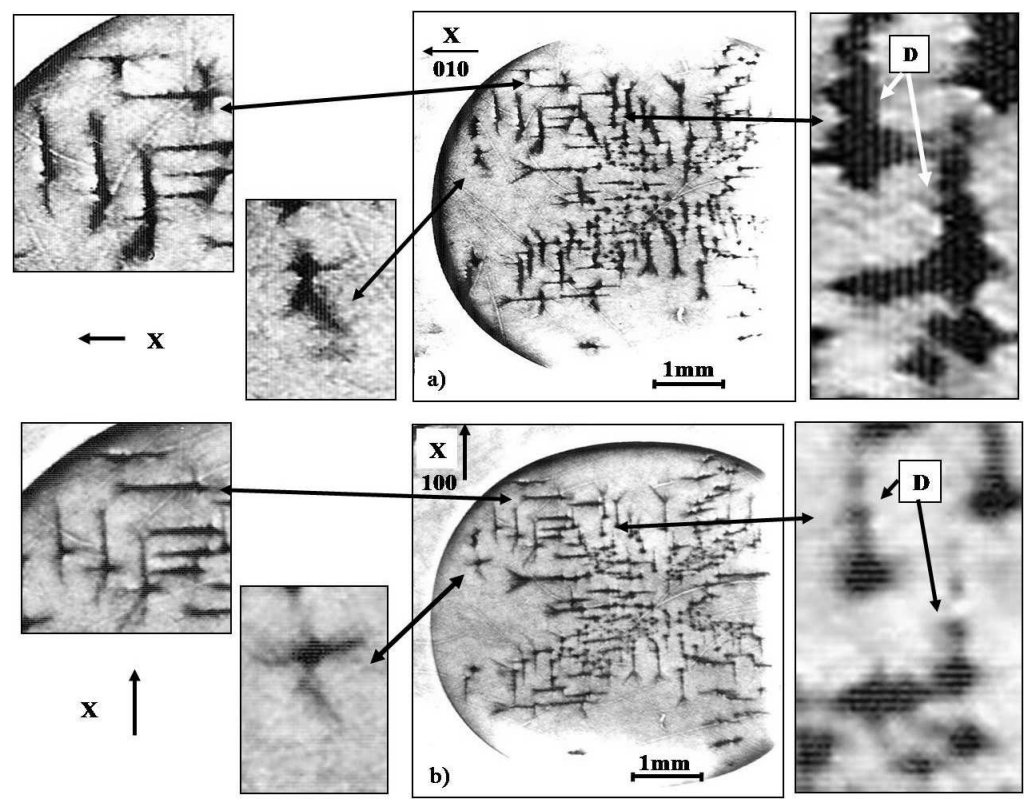

Fig. 2. Synchrotron radiation white beam back reflection projection topographs. Synchrotron radiation beam direction: (a) along [010], (b) along [100].

Owing to the high resolution of synchrotron radiation topographs, the "dotted" structure of the diffraction contrast can be distinguished (marked by D in Fig. 2a, b). It may be deduced that linear defects represent walls of elongated rod-like volume defects located in $\{100\}$-type lattice planes. The length of the rows depends on the number of rod-like defects arranged in the wall.

The points of intersection of individual defects with the crystal surface - the "dots" arranged in lines - may be observed in the optical micrograph (Fig. 3a). For comparison the X-ray topograph of the same crystal region is shown in Fig. 3b.

Additional information about the deformation of crystal lattice can be obtained by monochromatic wave SR back-reflection topography owing to its high sensitivity to small crystal lattice bending. The topographs of investigated sample were taken at angular positions of the crystal corresponding to half height of low-angle and high-angle flanks of the rocking curve. The corresponding images are reported in Fig. 4a, b. Comparing the images taken at low-angle and high-angle flanks of the rocking curve, one can notice the inversion of the diffraction contrast at the row of defects. It may be explained assuming that the lattice planes at both sides of the row are inclined in opposite directions.

\section{Conclusions}

The investigated sample exhibited a regular network of defects arranged in rows in $\langle 100\rangle$-type directions in the core region. The defects represent probably 

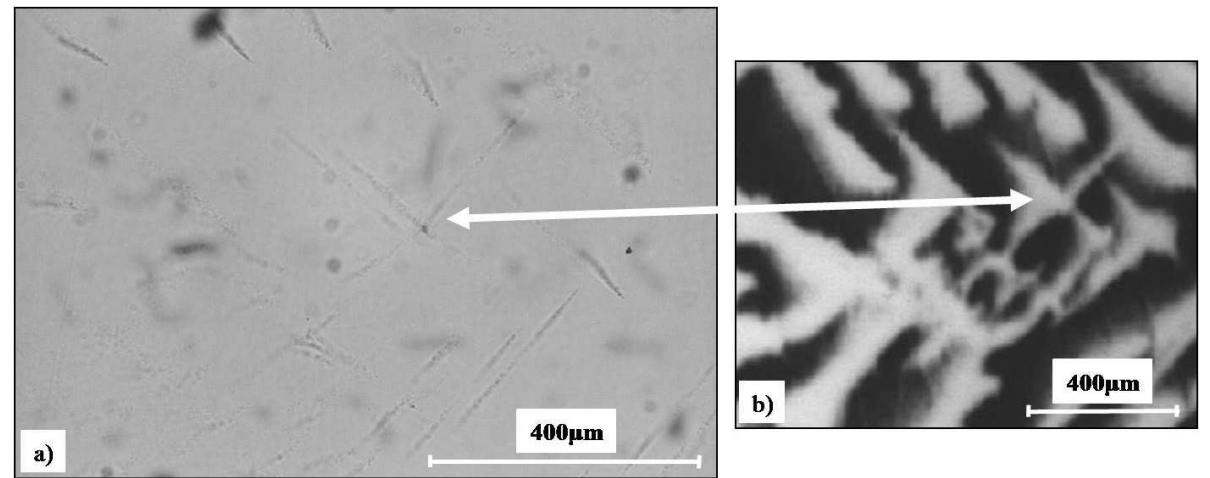

Fig. 3. (a) Optical micrograph (in transmission) of selected region of the sample core; (b) X-ray transmission projection topograph of the same region.

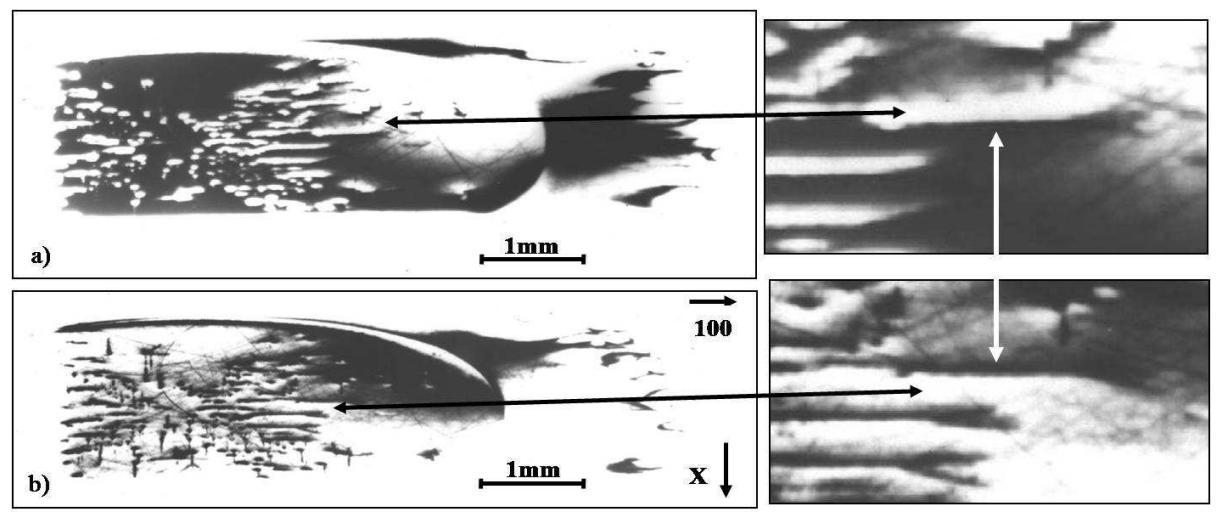

Fig. 4. Synchrotron monochromatic beam topographs, 008 reflection, $\lambda=0.115 \mathrm{~nm}$ : (a) low-angle rocking curve (RC) flank; (b) high-angle RC flank.

walls of elongated rod-like volume defects located in $\{100\}$-type lattice planes. It is possible that the defects can be some forms of dendritic shape, solute trails or kind of cellular structure and contain essentially the same phase crystallizing at a moment different than the rest of crystal. A probable reason of generation of these defects is the constitutional supercooling appearing during growth of compound crystals from non-congruent melts. It may produce instabilities of the crystal-melt interface causing local strong non-uniformity and breakdown of single crystalline growth [16-18]. The changes of chemical composition associated with observed defects will be checked in further studies.

\section{Acknowledgments}

The technical assistance of J. Bondziul is highly appreciated. This work was partly financed by the Ministry of Science and Higher Education, project no. N202 $01132 / 0609$ (2007-2008). 


\section{References}

[1] A. Pajączkowska, A. Gloubokov, Prog. Cryst. Growth Char. 36, 123 (1998).

[2] A. Pajączkowska, A. Kłos, D. Kasprowicz, M. Drozdowski, J. Cryst. Growth 198/199, 440 (1999).

[3] A. Novosselov, A. Kłos, E. Talik, A. Pajączkowska, J. Electron. Spectrosc. Relat. Phenom. 126, 129 (2003).

[4] K. Nakamura, R. Katano, L. Aoyama, Phase Transit. 42, 99 (1993).

[5] A. Dąbkowski, H.A. Dąbkowska, J.E. Greedan, J. Cryst. Growth 132, 205 (1993).

[6] R. Uecker, P. Reiche, S. Ganschow, D.C. Uecker, D. Schultze, Acta Phys. Pol. A 92, 23 (1997).

[7] A. Pajączkowska, A. Gloubokov, A. Kłos, C.F. Woensdregt, J. Cryst. Growth 171, 387 (1997).

[8] K. Wieteska, W. Wierzchowski, W. Graeff, M. Lefeld-Sosnowska, A. Pajączkowska, E. Wierzbicka, A. Malinowska, J. Alloys Comp. 401, 75 (2005).

[9] A. Malinowska, M. Lefeld-Sosnowska, A. Pajạczkowska, A. Kłos, J. Cryst. Growth 290, 149 (2006).

[10] K. Wieteska, W. Wierzchowski, W. Graeff, E. Wierzbicka, M. Lefeld-Sosnowska, A. Malinowska, HASYLAB Jahresbericht 2005, p. 401.

[11] K. Wieteska, A. Malinowska, M. Lefeld-Sosnowska, W. Wierzchowski, W. Graeff, E. Wierzbicka, HASYLAB Jahresbericht 2006, p. 633.

[12] A. Malinowska, M. Lefeld-Sosnowska, K. Wieteska, W. Wierzchowski, W. Graeff, in: Synchrotron Radiation in Natural Science, Bulletin of Polish Synchrotron Radiation Society, Vol. 5, No. 1-2, June 2006, Book of Abstracts of VIIIth Int. School and Symp. on Synchrotron Radiation in Natural Science, Zakopane (Poland) 2006, p. 75 , poster P-30.

[13] A. Malinowska, M. Lefeld-Sosnowska, K. Wieteska, W. Wierzchowski, W. Graeff, in: 8th Biennial Conf. on High Resolution X-Ray Diffraction and Imaging (XTOP 2006), Baden-Baden/Karlsruhe 2006, Book of Abstracts, p. 100, poster P160.

[14] A. Malinowska, M. Lefeld-Sosnowska, K. Wieteska, W. Wierzchowski, W. Graeff, A. Pajączkowska, in: 13th Int. Summer School on Crystal Growth (ISSCG-13), Park City, Utah (USA) 2007, Book of Abstracts, p. 42, poster P-M1.

[15] A. Malinowska, M. Lefeld-Sosnowska, K. Wieteska, W. Wierzchowski, A. Pajączkowska, W. Graeff, in preparation.

[16] D.T.J. Hurle, B. Cockayne, in: Characterization of Crystal Growth Defects by X-ray Methods, Eds. B.K. Tanner, D.K. Bowen, Plenum Press, New York 1980, p. 46.

[17] G. Müller, in: Perspectives on Inorganic, Organic, and Biological Crystal Growth: From Fundamentals to Applications, 13th Int. Summer School on Crystal Growth, Eds. M. Skowronski, J.J. De Yoreo, C.A. Wang, American Institute of Physics, Melville, New York 2007, p. 3.

[18] P. Rudolph, in Ref. [17], p. 69. 